\title{
A Review on Application of Artificial Intelligence in Teaching and Learning in Educational Contexts
}

\author{
Mehrnaz Fahimirad (Corresponding author) \\ Centre for R\&I for Tourism-SRI \\ Faculty of Social Sciences \& Leisure Management \\ Taylor's University Malaysia \\ 47500 Subang Jaya, Selangor, Malaysia \\ Sedigheh Shakib Kotamjani \\ Department of ELC, Shinas College of Technology \\ E-mail: sedigheh.shakib@shct.edu.om
}

Received: Sep. 12, 2018 Accepted: November 10, 2018 Published: December 15, 2018

doi:10.5296/ijld.v8i4.14057ＵRL: https://doi.org/10.5296/ijld.v8i4.14057

\begin{abstract}
Innovative educational technologies have revolutionized the methods of teaching and learning. Recently, with advancements of artificial intelligence, higher education has begun to adopt new technologies. This conceptual review paper aims to investigate the emergence of using artificial intelligence in teaching and learning in education. It examines the educational consequences of emergent technologies on how institutions teach and the way students learn. This study intends to predict the role of artificial intelligence in the future nature of education in a world. The effective application of artificial intelligence methods is considered as a means of improving the quality of teaching and learning. However, the challenges of integrating artificial intelligence in educational institutions is addressed. Moreover, the challenges faced by students in adopting artificial intelligence in terms of students' support, teaching, learning, and administration are discussed. This paper presents a concise overview of the most recent studies to showcase the application of artificial intelligence in educational contexts. The implications and directions for further research are suggested.
\end{abstract}

Keywords: Artificial intelligence, augmentation, higher education, machine learning, teaching, teacherbots 


\section{Introduction}

Higher education is fundamentally associated with advances in innovative technologies and high computational capacities of the intelligent machines. Hence, the developments in the field of artificial intelligence provides new opportunities and challenges for teaching and learning in the context of higher education; moreover, artificial intelligence has the potential to make effective changes in the core design of institutions in higher education. There is no ultimate definition regarding the concept of artificial intelligence among philosophers since Aristotle.

In 1950s, scientists started to investigate artificial intelligence solutions. The first solution to the problem of when a system is considered 'intelligent' was proposed by Turing (Russell \& Norvig, 2010). He suggested the simulated game to test the capacity of a human listener to distinguish between a dialogue with a machine and another human; if the system fails to detect this difference, we would admit having artificial intelligence (AI). Later in 1956, John McCarthy offered the most comprehensive definitions of artificial intelligence: "AI is the basis of the assumption that every aspect of learning or any other feature of intelligence can be described precisely the property of machine or program; the intelligence that the system demonstrates." (Kerr, 2017).

When it comes to the definition and application of artificial intelligence, most approaches only put emphasis on cognition and they just disregard other political, philosophical, and psychological aspects. The basic definition of artificial intelligence is based on the revision of past studies in the existing literature (Dacre Pool \& Qualter, 2012). Artificial intelligence (AI) is defined as computing systems, which engaged human-like processes such as learning, adapting, synthesizing, self-correction and the use of data for complex processing tasks.

Artificial intelligence is currently progressing at an accelerated pace, and this already impacts on the profound nature of services within higher education. For instance, Deakin University in Australia already applied IBM's supercomputer Watson as an emerging form of artificial intelligence and a solution to provide students with advice (Moles \& Wishart, 2016). This innovation significantly made efficient modification on the quality of services and workforce and dynamic time within the university. Hence, it should be pointed out that 'machine learning' is a growing research area in the field of artificial intelligence. Some artificial intelligence solutions are far dependent on programming, whereas some of these solutions have an integral capacity to make predictions and learn patterns. In this study, 'machine learning' is defined as a category of artificial intelligence (Schölkopf, 2015). Machine learning is software that make predictions, identify patterns, and apply those recently discovered patterns to circumstances that were not covered by their primary design.

The current review study aims to analyze the applications of artificial intelligence in teaching and learning. This conceptual review paper groups articles according to concepts and themes of integrating AI into education. It identifies the current 'understanding' of application of AI in current educational systems, discusses not only the advantages of AI applications but also the challenges of integrating AI into education. It provides a snapshot of future role of AI in educational system. 


\section{The Development of Artificial Intelligence in Education}

Artificial intelligence (AI) is defined as tools or instruments used extensively in different cities or campuses all over the world. They include some technologies like smart phones, internet, search engines, different apps, and household appliances. Artificial intelligence that everyone faces in everyday life, is the complicated set of software in the iPhone's Siri (Shulman \& Bostrom, 2012). Although it can be considered as AI with low complexity, it is labeled as an artificial intelligence project in America since 2001. In 2007, the apple company used this application in iPhone operation system. Nowadays, AI can be used by Google for its search engines. Moreover, AI is used in the engine, breaks, and navigation systems of all new cars. Self-driving technology is a top priority for development in some well-known companies like Volvo, Google, and Mercedes (Beam \& Kohane, 2016). This technology was first used on trial in Australia in 2015. Noticeably, self-driving technologies are using in mining corporation. In addition, self-driving trucks are used for different main reasons in Australia (Hillier, 2015).

To date, in higher education, students are placed in the center of challenges for learning and teaching. Human-AI interaction is considered as a kind of solution or collaboration that can assist the disabled people around the world. Therefore, these technologies can inspire the people to take advantage of AI in higher education. It can motivate learners and teachers to be more engaged in the learning and teaching process. In his Encyclopedia of Science, Technology and Ethics, (Kelley et al., 2016) refers to a cyborg as "a crossbreed of a human and a machine". Based on (Programas de las Naciones Unidas para el Desarrollo, 2015), complex-computing systems that use the machine learning algorithms are able to help individuals with different kinds of abilities. Moreover, to some degree, they are involved in some human-like processes, and they can do some complicated tasks in the world of teaching and learning. Thus, a new era for higher education is opened for institutions.

This kind of interaction between human and machine is a turning point to help human beings learn and memorize information. However, a main question has been unresolved on how long does it take to this type of interface (AI) to increase the level of memory and cognition among the human beings. According to MIT scholars there will be some changes in technologies applied in education especially after 2007 when the first models of iPhone came into the market. Not only iPhone is a new technology that assists us to access and use information that was not possible a few years ago, but also it causes an influential cultural shift that influences the individuals' social lives (Liebowitz, 2001). However, if the emphasis of "cyborgs" shifts from science fiction to the computer application for both teachers and learners, 'cross- breeds' or cyborgs of human and machines can be manifested in the world of education in near future.

It is possible to witness the effect of artificial intelligence on economy that has drawn the attentions of numerous analysts. In 2014, in the European Union, Google had the largest investment ( $\$ 400$ million) in the acquisition of Deep Mind technologies. Today, DeepMind Technologies as Google DeepMind is considered as a startup of London-based artificial intelligence, which can be used in machine learning. Remarkably, Google had another investment on Artificial Intelligence in the German Research Centre (Lanctot et al., 2017). Based on the report by their website, this research center was the leading research center 
around the world on Artificial Intelligence and its application (Avramidis, Popović, \& Burchardt, 2015). The field of artificial intelligence is an area that is competed by some giant technologies such as Apple, Google, Microsoft, and Facebook, and they invest a lot on its new applications and research. According to a report by Google in December 2015, a new model of computer that was called D-Wave $2 \mathrm{X}$ is able to do the complex operations of AI (Caplar, Tacchella, \& Birrer, 2017). This model of computer is faster than the current computers. To Google researchers, it is a leap ahead and significant breakthrough in the area of AI. "We hope it helps researchers construct more efficient and more accurate models for everything from speech recognition, to web search, and protein folding" (Elsayed, Thomas, Marriott, Piantadosi, \& Smith, 2015).

Investing on AI can influence on academic settings. Perhaps the economic problems for students of higher education are good reasons for seeking the AI solutions. As indicated in the previous examples, the combination of a machine and human brain is feasible, and this issue poses a challenge for the teachers to look for new dimensions, functions, and pedagogies in many different contexts for learning and teaching. For instance, the interface of brain and computer has drawn the attentions of researchers in all around the world. By taking the analysis methods and brain signals together with some approaches in new computing systems, the professionals in the field of computer have provided some solutions in order to manage the software with an interface of brain and computer (Kena et al., 2015). Brain-computer interface can take and decode the brain activity. In addition, it can make the communication easier among those have disabilities associated with motor function (Pandarinath et al., 2017). Our skills and abilities are enhanced by quick expanding of technology to use AI functions. As Schleicher pointed out, "Innovation in education is not just a matter of putting more technology into more classrooms; it is about changing approaches to teaching so that students acquire the skills they need to thrive in competitive global economies"(Schleicher, 2012).

Artificial Intelligence (AI) approaches has the ability to develop and imitate the decision-making process which are adopted by people. Different AI techniques have been using in adaptive educational systems. These techniques can be referred to Fuzzy Logic, Neural Networks, Decision Trees, Bayesian Networks, Hidden Markov Models, Genetic Algorithms. However, there is no consensus regarding the creation of standard approach so far to figure out which technique has the most suitable learning theory of AI to apply for a particular learning environment. Moreover, scientists have not developed software tool yet to facilitate determining the learning style from students' learning behavior. What is required in learning and teaching is a tool to easily configurable and accessible in various learning environments, such as traditional or eLearning.

In educational system, the educational goals can be better achieved and managed by artificial intelligence (AI). By using AI instructors can analyze students in a class and recognize who is a slow learner to understand the topics. If student has some weaknesses in some areas or he/she fails to understand few topics consequently AI analysis would showcase this report to lecturer or parents then appropriate action can be taken by lecturer to scaffold learning.

Furthermore, Artificial Intelligence has the potential to draw attention to which topics of 
courses need to be improved, since lecturers might not always be cognizant of gaps in their educational materials which can lead to students' confusion. Classes can be tailored according to students' profile and students' interests can be stimulated by exposing them to various content and courses. Moreover, AI can assist lecturers in terms of homework. For an instance, Coursera which acts as an enormous open online course provider, evaluate students' responses to a problem. As Coursera found that a large number of students submitted the incorrect answer to a homework, the teacher is alerted through the system and the system gives prospective students a message to offer clues to the right answer. This AI system contributes to provide explanation of courses, and to ensure that all students are building the same conceptual basis.

Hence, lecturers with the ability to employ technology for their teaching strategy can gain the advantages of automatic data created from students' engagement. Since these digital information systems are prone to store and process huge amounts of information quickly in a short time. Apart from these specialized algorithms, these systems could identify the level of students' engagement and make sense of their behavioral patterns that appear in a teacher's class and could inform these results to the teacher. The application of this technology assist lecturers to manage their classes appropriately and they can spend less time on paperwork and spend more time on obtaining invaluable insights from AI tools to deliver higher quality research-informed teaching. In this paper, only we have explained just a few tools that will shape the application of AI in educational contexts in the future.

\subsection{Artificial Intelligence Can Automate Grading in Educational Context}

Grading homework and tests usually takes a significant amount of time. This time could be used to work on professional development, interact with students and prepare for class. As AI might not truly replace human grading, it's about to replace human grading. AI automated grading is able to grade nearly fill-in-the-blank and all kinds of multiple choice testing. However, essay-grading software is still in its early stages yet and it will be improved over the coming years.

\subsection{Students Could Receive Supplementary Support From AI Tutors}

It is obvious that human tutors can teach some subjects that machines are not able to teach; however, students will be tutored by Artificial intelligence machines. Currently, there are some tutoring programs which are based on artificial intelligence to help students through writing, basic mathematics, and other subjects.

These AI programs can instruct students only basics subjects; however, these machines aren't perfect to tech high-order thinking and creativity to students. With the rapid advancement of technology, advanced tutoring systems might not be an unattainable dream.

\subsection{AI programs Can Give Students and Lecturers a Constructive Feedback}

AI has the potential to provide feedback to lecturers and students about the success of the course. Some schools employ AI systems to track and monitor students' progress and to notify professors if there is an issue with students' performance. Moreover, these AI systems provide 
students with the appropriate support and provide professors with feedback to improve the instruction in the related subject matter.

\subsection{AI Can Alter the Role of Teachers}

As it mentioned before, AI able to take charge of various tasks such as grading, giving constructive feedback on students' performance. Furthermore, they even might be a substitute for teaching. They could be programmed to provide knowledge, to ask questions and find information for very basic course materials. However, in several cases of application of AI in education, AI has shifted the role of the teacher to a facilitator. Teachers can integrate AI lessons as supplementary materials to assist weak students and provide hands-on experiences in the form of human interaction for students.

\subsection{AI Makes the Process of Trial-and-Error Learning Less Daunting}

AI systems have been designed to assist students in the process of learning, in this context, trial and error process is much less intimidating to students. Since AI systems provide students with fairly judgment-free environment of learning, moreover, as AI tutors can suggest solutions to students' performance. Indeed, AI is considered as an optimum system for learning, because AI itself frequently learn through a trial-and-error method.

Finally, AI systems may change the way students learn, and assist them to develop basic skills. In fact, recently artificial intelligence is about to change fundamentally the process of learning in educational contexts. AI programs are substituting particular types of classroom instruction by providing support for students to learn from anywhere in the world at any time. In addition, in future, AI system might replace lecturers in some subjects. Currently, some educational programs are equipped with AI, scaffolding students to learn basic skills. However, as AI program developers are advancing, AI will more likely provide students with extensive range of services.

AI systems provide this level of insight to not only save time, but also can provide teachers with more details which may not be obvious or possible for teachers to identify them. Classroom AI systems have a high capability to analyze multiple sources of data and compare those data to known patterns. They can recognize the source for problems and also give guidance to lecturers to achieve more consistent outcomes across various classes.

\section{Challenges of Artificial Intelligence Solutions}

Based on in depth literature review, we have found the following challenges in implementing AI systems into educational system:

Cost: Provision of the initial outlays for software and cloud support are very costly for educational systems. In addition, not only the costs for the continuous employees training is expensive but also on-going training of the AI system would be costly if organizational processes change.

Culture clash: The organizations might have considered any changes as suspicious. Since there are several technology options; therefore, it is difficult decision to restrict the potential options 
and the most appropriate implementation path.

The application of AI has been a very attractive solution in education educational systems. In addition, there are some other reasons for the application of AI like the great number of students, and broader financial pressures. Thus, these kinds of factors make the universities as markets, which can attract a large number of students and increase the enrolment numbers. However, one major problem was with the lectures to cope with a large number of students studying from different time zones (Various, 2014). Another problem of the lecturers was with the students who were at different rates of progress, or different fundamental skills for the courses they have taken. The way how to help students to increase their learning activities to obtain desired results, do the assessment, and give constructive feedback have been remained as unresolved issues. The observation in Teacherbot was made by Sian Bayne revealed that Interventions in Automated Teaching, "are driven by a productivity oriented solutionism," not by pedagogical reasons. (Ferguson et al., 2015).

One lesson that deserves attention is MOOC. In 2008, MOOCs were used for the first time, after that time we heard about changes that came over the higher education. The outcomes of research on this issue revealed the failure of MOOCs to meet the participants' promises. However, an important issue refers to some irrational and unreserved ideas surrounded MOOCs when the decision-makers failed to consider the key principles like evidence-based arguments or academic skepticism. Moreover, they were not interested in learning and they were just thinking about the financial profits (Popenici, 2013).

In fact, online learning can effectively help higher education to come to some important aims in teaching, learning and conducting the research. But unfortunately, in MOOCs a limited concentration is on just a technology solution without considering the arguments that leads to some distractions in education. Educational system is taking advantage of AI in the area of teaching, learning. Accordingly, recent development in non-invasive brain-computer interfaces made us rethink about the role of teachers, or they did their best to replace teacher-robots, or virtual "teacherbots instead of human teachers (Hayes, 2015). In this way, some solutions are suggested to apply brain computer interface (BCI) devices which can make it possible for the students to fully focus on the learning tasks and content (Beam \& Kohane, 2016). In the same way, super-computers like IBM's Watson are able to check automatically the presence of teachers during the whole semester. The possibility of communicating and commanding through the broader applications of AI in the area of learning and teaching indicates a serious revolution in technology that can extensively convert the structure of educational system. Fully online courses can be run by taking advantage of personalized learning with a teacherbot. For the administrative part of teaching, Teacherbots computing solutions are used to mainly deal with content delivery, basic feedback and supervision, which can be disruptive for traditional teaching assistants (Kena et al., 2015).

There are numerous applications for $\mathrm{AI}$ as a computer helps as a tutor and also it manages learning among the students. However, it is worrying that AI automated solution is used instead of teaching which is a superficial, but profitable approach. We are at the era that we are looking for a new pedagogical philosophy that assists learners obtain some essential skills for 
social life. Graduates are supposed to work in situations full of conflicts, without any sufficient information, and with high rate of risks and uncertainty (Gal, 2016). Along with being exposed to immense applications of technology, rethinking about the application of technology in education seems necessary. It is correct that AI solutions are advanced; however, it is impossible to imagine they can replace the human mind. Although there are some optimistic views, the current developments in technology seem unlikely to happen in future. According to RuchirPuri, "There is a lot of hype around AI, but what it can't do is very big right now. What it can do is very small." It is an encouragement for policy makers to review the educational systems to have new paradigms and focus on creativity, and civic engagement.

With considering the role of AI in guiding the students' learning, and monitor their participation, it is able to feed information and materials based on the students' needs. In addition, it can provide them with feedback and encouragement. However, by taking advantage of this technology, the teachers make the students ready for hyper complexity. Thus, in future employability is not the only aim of using technologies (Ratliff, Bradley, Bagnell, \& Chestnutt, 2006). Although teacherbots are disruptive to traditional teaching staff, knowing how to apply them seems essential for the benefits of the students (Mason, 2010).

In 2017, the people had little information about the teacherbot and its capabilities. However, in near future AI technology will be at the backdoor of our lives and will focus the educational systems (Ferguson et al., 2015). AI solutions have capabilities to monitor our movements, choices, gauging weaknesses and strengths, providing feedback, comparative analytics, encouragement, and predictive text. Altogether, a teacherbot is considered as a complex algorithmic interface, and it can take advantage of artificial intelligence for personalized education. In addition, a teachrbot can provide the content; it will also be like a supervision or guidance for the students. Thus, it can help the teachers. Teacherbots refer to any kind of machine-based hardware or software, which can play the role of a teacher to organize teaching materials and provide quick responses to a range of questions. They have a potential to facilitate, monitor, assess, and manage students' learning during the online learning(Siân Bayne and Jen Ross, 2016). These explanations and solutions are more feasible than what many universities think. Those teachers who transmit the information to passive students with the old systems in a class or in front of a computer are confronted with an affordable and very personalized alternative of AI solutions like "Jill Watson"(Bayne, 2008). Although personal guidance and contact time by faculty members are considered as an advantage in the higher education to provide the quality education, hence the intelligent machines are more likely to meet the students' learning needs.

\section{Results and Discussion}

Since artificial intelligence solutions have potentiality to transform university administrative services, teaching and learning may experience a totally a various range of challenges. To Perez (2016), AI solutions can do the automated tasks. However, it is not imaginable that they can do the more complicated tasks of higher learning (Soto et al., 2012). The current paper indicates some evidences of AI application technologies for enhancement of teaching and learning in educational system. However, we face with some limitations in the application of 
technology; AI cannot be a replacement for teachers. Today, computing algorithms can influence on every aspects of human lives from the peoples' credit scores to employability. To date, higher education will be at the heart of this intense transformation, and can cause remarkable opportunities and risks. This essential issue needs attention and analysis from the academic points of view. Thus, in the area of teaching, we should look for solutions and technological progress will be a common solution (Bengio et al., 2013).

The application of technology in higher education is suitable when it increases the opportunities and capabilities of research, teaching and learning. The purpose of this study is supported with the purposes of other past research in this area like the one entitled as "National Artificial Intelligence Research and Development Strategic Plan". The Report stated AI increased the human capabilities, and the walls between humans and artificial intelligence were slowly collapsed (National Research Council, 2016).

In fact, the development of machine learning and AI in institutions of higher education has made some possibilities and challenges. However, it is essential to consider that education is not a technology centric solution, but a kind of human-centric effort. In spite of a quick advancement in AI, solely dependency on technology is not correct. Therefore, it is essential to focus on this idea that human beings themselves should recognize the problems and risks. In addition, they should ask some important questions about privacy, and control of fostering creativity requirement. Moreover, they should leave the door open for serendipity paths in the process of learning and teaching (Council, 2011). The motive for AI is like a panacea in higher learning and leaves those who are in the path under the wheels of reality. However, an important issue in education is maintaining academic skepticism. Generally speaking, we need to consider this aim to nurture responsible citizens and educated minds.

Enhancing human thinking and increasing the teaching and learning process is the main aim of technology in education. With the increasing application of AI, it is essential for academic institutions to be informed of whether controlling over algorithms is not monopolized by tech-lords. To Pasquale (2015), making decisions should be made automatically based on human reflection (Secundo, Vecchio, \& Passiante, 2015). The quick advancements of AI can be doubled by universities when they try to look for some economic solutions to stabilize the budgets. In education, there is an ability to change a number of teaching assistants and administrative staff. Thus, exploring the impacts of these factors on learning is very essential. These impacts will be profound especially in contexts that there are demands for initiative and creativity for graduates.

The current conceptual review paper aims to investigate the application of artificial intelligence in teaching and learning. This study also tries to analyze the past studies on how artificial intelligence can modify the way students learn in higher education institutions.

\section{Conclusion}

Increasing the application of AI is not the reason to disregard the profound debate about the role of teaching and learning in education. The advancement of technology and job displacement admitted extensively, however, it indicates that teachers' role should be 
reconsidered for teaching in education.

Taking advantage of AI or IT solutions to recognize plagiarism can raise this question that who is responsible for teaching and learning. In addition, because of complex algorithms that can transfer their own biases in operating systems, AI software has the potential to replace a range of tasks, which are at the center of teaching practice in higher education (Rajasingham, 2009). Nowadays, the models of pedagogies and their relations with AI are rethought by universities. Moreover, institutions of higher education can anticipate the possibilities and challenges that was a chance to accept artificial intelligence in teaching and learning (Drigas \& Ioannidou, 2013). These solutions make opportunities for teaching and learning, while supporting learning can keep the wholeness of central values and the aim of higher education. Finally, there is a requirement for conducting research on the applications and improvement of artificial intelligence and the possibility of increasing the human knowledge. Finally, it is essential to concentrate on the new role, the teachers play on new learning by the students, insisting on some factors like creativity, imagination, innovation and skills which can scarcely be performed by machines.

\section{Acknowledgement}

\section{Funding}

This research is not funded by a specific project grant.

\section{Conflict Exists}

The Author's declares that there is no conflict of interest regarding the publication of this article.

\section{Ethical Approval}

This article does not contain any studies with human participants or animals performed by any of the authors.

\section{References}

Bayne, S. (2008). Higher education as a visual practice: Seeing through the virtual learning environment. Teaching in Higher Education. https://doi.org/10.1080/13562510802169665

Beam, A. L., \& Kohane, I. S. (2016). Translating artificial intelligence into clinical care. JAMA - Journal of the American Medical Association. https://doi.org/10.1001/jama.2016.17217

Bengio, Y., Courville, A., Vincent, P., Seliya, N., Wald, R., Muharemagic, E., ... Mu, J. (2013). Representation Learning: A Review and New Perspectives. IEEE Transactions on Pattern Analysis and Machine Intelligence. https://doi.org/10.1109/TPAMI.2013.50

Billings, M. E., Lazarus, M. E., Wenrich, M., Curtis, J. R., \& Engelberg, R. A. (2011). The Effect of the Hidden Curriculum on Resident Burnout and Cynicism. Journal of Graduate Medical Education. https://doi.org/10.4300/JGME-D-11-00044.1 
Caplar, N., Tacchella, S., \& Birrer, S. (2017). Quantitative evaluation of gender bias in astronomical publications from citation counts. Nature Astronomy. https://doi.org/10.1038/s41550-017-0141

Council, N. R. (2011). SucceSSful K- 12 identifying effective approaches in Science. Social Sciences.

Dacre Pool, L., \& Qualter, P. (2012). Improving emotional intelligence and emotional self-efficacy through a teaching intervention for university students. Learning and Individual Differences. https://doi.org/10.1016/j.lindif.2012.01.010

Drigas, A. S., \& Ioannidou, R. E. (2013). A Review on Artificial Intelligence in Special Education. In Communications in Computer and Information Science. https://doi.org/10.1007/978-3-642-35879-1_46

Elsayed, N., Thomas, B., Marriott, K., Piantadosi, J., \& Smith, R. (2015). Situated Analytics. In 2015 Big Data Visual Analytics, BDVA 2015. https://doi.org/10.1109/BDVA.2015.7314302

Ferguson, R., Clow, D., Beale, R., Cooper, A. J., Morris, N., Bayne, S., \& Woodgate, A. (2015). Moving through MOOCS: Pedagogy, learning design and Patterns of Engagement. In Lecture Notes in Computer Science (including subseries Lecture Notes in Artificial Intelligence and Lecture Notes in Bioinformatics). https://doi.org/10.1007/978-3-319-24258-3_6

Gal, Y. (2016). Uncertainty in Deep Learning. PhD Thesis. https://doi.org/10.1371/journal.pcbi.1005062

Hayes, S. (2015). MOOCs and Quality: A Review of the Recent Literature. QAA MOOCs Network.

Hillier, J. (2015). Performances and performativities of resilience. In Evolutionary Governance Theory: Theory and Applications. https://doi.org/10.1007/978-3-319-12274-8_12

Kelley, T. R., Knowles, J. G., Abell, S., Lederman, N., Bandura, A., Bandura, A., ... Williams, D. (2016). A conceptual framework for integrated STEM education. International Journal of STEM Education. https://doi.org/10.1186/s40594-016-0046-z

Kena, G., Musu-Gillette, L., Robinson, J., Wang, X., Rathbun, A., Zhang, J., ... Velez, E. D. (2015). The Condition of Education 2015. U.S. Department of Education, National Center for Education Statistics. https://doi.org/10.4135/9781412963916.n394

Kerr, S. (2017). Exploring the impact of artificial intelligence on teaching and learning in higher education. https://doi.org/10.1186/s41039-017-0062-8

Lanctot, M., Lanctot@, D., Zambaldi, V., Vzambaldi@, D., Unas, A. ${ }^{-}$, Deepmind, G.,... Tuyls, K. (2017). A Unified Game-Theoretic Approach to Multiagent Reinforcement Learning. In 31 st Conference on Neural Information Processing Systems (NIPS 2017).

Liebowitz, J. (2001). Knowledge management and its link to artificial intelligence. Expert 
Systems with Applications. https://doi.org/10.1016/S0957-4174(00)00044-0

Martin, F. G. (2012). Will massive open online courses change how we teach? Communications of the ACM. https://doi.org/10.1145/2240236.2240246

Mason, M. (2010). Sample Size and Saturation in PhD Studies Using Qualitative Interviews. Forum Qualitative Sozialforschung / Forum: Qualitative Social Research. https://doi.org/ISSN 1438-5627

Moles, J. j moles deakin edu au, \& Wishart, L. llewwllyn wishart deakin edu au. (2016). Reading the Map: Locating and Navigating the Academic Skills Development of Pre-Service Teachers. Journal of University Teaching \& Learning Practice.

National Research Council. (2011). A Framework for K-12 Science Education: Practices, Crosscutting Concepts, and Core Ideas. Social Sciences.

Pandarinath, C., Nuyujukian, P., Blabe, C. H., Sorice, B. L., Saab, J., Willett, F. R., ... Henderson, J. M. (2017). High performance communication by people with paralysis using an intracortical brain-computer interface. ELife. https://doi.org/10.7554/eLife.18554

Popenici, S. (2013). Higher Education and Affluenza. Popenici.

Programas de las Naciones Unidas para el Desarrollo. (2015). Human Development Report 2015. Work for Human Development. Undp. https://doi.org/ISBN: 978-92-1-126398-5

Rajasingham, L. (2009). The Impact of Artificial Intelligence Systems on Future University Paradigms. Journal of Online Learning and Teaching. https://doi.org/https://dx.doi.org/10.1108/17506200710779521

Ratliff, N. D., Bradley, D., Bagnell, J. A., \& Chestnutt, J. (2006). Boosting Structured Prediction for Imitation Learning. Advances in Neural Information Processing Systems.

Russell, S. J., \& Norvig, P. (2010). Artificial Intelligence: A Modern Approach. Artificial Intelligence. https://doi.org/10.1017/S0269888900007724

Schleicher, A. (2012). Preparing Teachers and Developing School Leaders for the 21st Century: Lessons from Around the World. OECD Education and Skills. https://doi.org/10.1787/9789264174559-en

Schölkopf, B. (2015). Artificial intelligence: Learning to see and act. Nature. https://doi.org/10.1038/518486a

Secundo, G., Vecchio, P. Del, \& Passiante, G. (2015). Creating innovative entrepreneurial mindsets as a lever for knowledge-based regional development. International Journal of Knowledge-Based Development. https://doi.org/10.1504/IJKBD.2015.074301

Shulman, C., \& Bostrom, N. (2012). How hard is artificial intelligence? Evolutionary arguments and selection effects. Journal of Consciousness Studies.

Siân Bayne and Jen Ross. (2016). Manifesto Redux: making a teaching philosophy from networked learning research. Proceedings of the 10 Th International Conference on 


\section{Macrothink}

International Journal of Learning and Development

Networked Learning 2016.

Soto, J. A., Armenta, B. E., Perez, C. R., Zamboanga, B. L., Umaña-Taylor, A. J., Lee, R. M., ... Ham, L. S. (2012). Strength in numbers? Cognitive reappraisal tendencies and psychological functioning among Latinos in the context of oppression. Cultural Diversity and Ethnic Minority Psychology. https://doi.org/10.1037/a0029781

\section{Copyright Disclaimer}

Copyright for this article is retained by the author(s), with first publication rights granted to the journal.

This is an open-access article distributed under the terms and conditions of the Creative Commons Attribution license (http://creativecommons.org/licenses/by/4.0/). 